\title{
Evaluation and treatment of postconcussive symptoms
}

\author{
Thomas W. McAllister ${ }^{\mathrm{a}, *}$ and David Arciniegas ${ }^{\mathrm{b}}$ \\ ${ }^{a}$ Dartmouth Hitchcock Medical Center, Lebanon, NH 03756, USA \\ ${ }^{\mathrm{b}}$ Denver Veterans Affairs Medical Center and University of Colorado Health Sciences Center, Denver, CO, USA
}

\begin{abstract}
Postconcussive symptoms such as headache, dizziness, irritability, and difficulties with memory and attention are reported frequently after traumatic brain injuries (TBI) of all severities. The etiology of these symptoms in individuals with mild TBI has been a subject of some controversy with theories ranging from neural damage to malingering. Furthermore, although the term postconcussive syndrome is commonly used clinically and in the scientific literature, it is not clear that postconcussive symptoms constitute a syndrome per se. Instead, it may be the case that the various symptoms that commonly co-occur after TBI are relatively independent consequences of a single neurological event. In other words, because the locations and severity of injury vary between individuals despite ostensibly similar injuries, it follows that there should be variations in symptom type and severity between individuals as well. This article reviews the sequelae and natural course of recovery from mild TBI, the evidence regarding both persistent postconcussive symptoms and the postconcussive syndrome, and outlines an approach to the assessment and treatment of individuals with these symptoms after TBI.
\end{abstract}

Keywords: Postconcussion syndrome, mild traumatic brain injury, postconcussive symptoms

\section{Introduction}

The nature, etiology, and treatment of symptoms after mild traumatic brain injury (MTBI) remains one of the most controversial areas in brain injury rehabilitation. Discussions of MTBI and its consequences are complicated by this controversy, much of which is borne out of medicolegal biases, methodological problems in scientific studies, and conceptual disagreements in this area. For example, there is a longstanding, and probably ill-founded, view that because MTBI is uniformly associated with a good recovery, individuals with a poor outcome are to be considered aberrant and regarded with some suspicion [1]. However, arguments to the contrary of this traditional view must account for the observations that complex motivations

*Address for correspondence: Thomas W. McAllister, M.D., Professor of Psychiatry, Dartmouth Medical School, Dept of Psychiatry, Dartmouth Hitchcock Medical Center, One Medical Center Drive, Lebanon, NH 03756, USA. E-mail: Thomas.W.McAllister@ Dartmouth.edu. prompted by litigation or compensation proceedings may, in some individuals, influence the degree of reported symptomatic distress [2-4] and that some individuals do intentionally exaggerate or feign symptoms [5]. Further complicating such discussions is the legacy of many early studies in this area that failed to account for the lack of specificity of post-TBI complaints and related distress, the relationship between injury to other bodily systems and "postconcussive" symptoms, and the high base rates of such symptoms in the general population [6,7] and in other populations [8-10], leaving uncertain the significance of such symptoms after TBI.

There are also conceptual disagreements about the framework within which the legitimate distress and disability that some individuals experience after MTBI should be considered. Some experts regard the common signs and symptoms after brain injury as a "postconcussive syndrome" that reflects a neurobiology and pathogenic process specific to TBI. Others regard the elements of this "syndrome" as merely co-occurring symptoms seen commonly after brain injury, each ini- 
tiated by the same event (TBI) but produced by different underlying mechanisms. These different perspectives on the nature of postconcussive problems entail substantive differences in the approach to evaluation and treatment of so-affected patients, with the syndromic perspective suggesting a unified and an appealingly simple view of underlying pathogenesis, neurobiology, and treatment, and the symptomatic perspective entailing a relatively more complex understanding of the relationship between symptoms, brain-behavior relationships, neuropathologies, and treatments.

The scientific literature is therefore rife with arguments regarding the nature of such symptoms after MTBI, including disagreements about the relative contributions of neural injury, secondary gains, expectational sets, and/or co-morbid psychiatric disorders to the development of acute and chronic postconcussive problems. There is within these arguments a theme parallel to the "organic vs. functional" arguments made about the etiologies of many neuropsychiatric conditions that, like the postconcussive syndrome, are complex and difficult to understand. It is worth noting that the modern-day form of this argument, in which "organic" denotes conditions resulting from "real" brain pathology and "functional" refers to problems with a "psychological" basis, is not an accurate application of the terms as originally suggested by Coombe (17971847); these distinctions were originally intended to permit distinction between those conditions arising from localized pathology (i.e., organic) and those resulting from disturbances in the function of the brain as a whole (hence, functional). With regard to postconcussive symptoms or the postconcussive syndrome, the modern-day form of this argument is alternately used to argue for a neurobiological basis of such problems or instead to ascribe the entirety of such to psychological or motivational factors.

Such arguments do a serious disservice to clinicians, scientists, and patients regardless of the context in which they arise. They establish unnecessary barriers to fully integrating and hence understanding the nature of such symptoms in specific individuals, the nature of MTBI and postconcussive problems more generally, and delivery of appropriate evaluation, treatment, and other needed support. Additionally, they provide a means by which some unscrupulous parties will seek to impugn the veracity of any and all reported postconcussive symptoms in order advance their own financial interests. However, before asserting a strong position regarding the nature of these symptoms - whether they are products of neurobiological dysfunction, psycho- logical or motivational factors, or some combination thereof - it is important first to consider carefully what is known about the pathophysiology of mild TBI, the natural history of mild TBI, and the types of symptoms in question and the extent to which their presentation and response to treatment supports the construct of a true postconcussive syndrome.

\section{Pathophysiology of mild TBI}

Brain injury occurs along a continuum of severity. Although postconcussive symptoms or the postconcussive syndrome are most often written about in the context of milder degrees of brain injury, the terms are not synonymous with MTBI [11,12]. Different schemes have been proposed for categorizing injury severity but there is no universally accepted definition of MTBI. Injuries in which loss of consciousness (LOC) is less than 30 minutes and in which Glasgow Coma Scale (GCS) [13] scores are 13 or greater are usually considered consistent with mild brain injury. More recently, the special task force of the American Congress of Rehabilitative Medicine proposed a definition of mild traumatic brain injury which is largely consistent with the above clinical traditions, while also extending the concept of mild injury to include alteration of consciousness manifested by incomplete memory of the event (posttraumatic amnesia, or PTA) and/or being "dazed and confused" even where there is no frank loss of consciousness [14]. When such deficits are prolonged (i.e., LOC $>30$ minutes, PTA $>24$ hours, etc.), the injury is considered to be of more than mild severity. After an injury of any severity, however, a litany of symptoms such as headache, dizziness, sleep disturbance, cognitive impairment, irritability or affective lability, and aggression may develop to varying degrees. Thus mild TBI refers only to initial injury severity, whereas postconcussive symptoms or the postconcussive syndrome denote a constellation of signs and symptoms that may be reported after a TBI of any severity [15-18].

Even brief alterations in level of consciousness are associated with widespread neuronal dysfunction [19, 20], and there is evidence that structural neuronal damage may result from even very mild brain injury [21, 22]. For example, Oppenheimer [21] found evidence of axonal injury in five patients with minor or trivial injuries. Using immunostaining for amyloid precursor protein as a marker for axonal injury, Blumbergs et al. [22] reported multifocal axonal injury in five in- 
dividuals who had sustained very mild injuries with periods of unconsciousness as short as one minute.

Animal models of brain injury using the fluid percussion model in cats [23] and controlled angular acceleration devices in nonhuman primates [24] strongly suggest that even mild brain injury is associated with evidence of axonal injury. Although axotomy may occur at the time of injury, delayed axotomy contributes significantly to the neuropathological outcome. Delayed axotomy is believed to occur subsequent to initial changes in the permeability of the axolemma (axonal membrane) and disruption of certain elements of the cytoskeleton, particularly axonal neurofilaments. This in turn can lead to axonal distortion and disruption of axoplasmic transport (see Povlishock and Christman for review [25]). Secondary deafferentiation (structural changes and sometimes neuronal death due to loss of synaptic input) in target areas of the afflicted axon can follow thereafter [23].

Williams et al. [26] studied 155 consecutive patients with mild brain injury, and found that 32 had parenchymal contusions or hemorrhages (20\%), and 27 (17\%) had subdural or epidural hematomas despite the ostensibly "mild" mechanism of injury. Three recent large cohort studies [27-29] in mild TBI patients with GCS scores of 15 , representing over 4,000 patients, found abnormal CT scans in $5-10 \%$ of this group. In general, individuals with GCS scores of 13 or 14 have a higher frequency (20-35\%) of abnormal findings on CT scans [30-33]. Furthermore, the presence of structural lesions in mild TBI, whether on CT or magnetic resonance imaging (MRI), is associated with outcomes more consistent with those seen in moderate TBI [26, $34]$, at least from a cognitive standpoint. Other neuropsychiatric outcome indices may be more closely associated with the duration of PTA and less closely associated with GCS scores [35].

The above suggests that brain injuries that might be considered trivial based solely on the degree and duration of altered consciousness are, contrary to some expectations, in many cases relatively severe neuropathologically. The effects of mild injuries, both macroscopic and microscopic, are similar in quality and location to those seen with moderate and severe degrees of brain injury, again supporting the concept of continua in both injury severity and sequelae. This theme is echoed in several recent neurodiagnostic studies in this population, particularly those employing newer neuroimaging and electrophysiological techniques, the results of which may offer additional insights into the neurobiological substrates of postconcussive symptoms.

\subsection{Neuroimaging}

A variety of MRI based techniques have been introduced over the last several years that enhance the ability to detect subtle sequelae of traumatic brain injuries. Most of these techniques manipulate the image acquisition parameters or use various pre-pulses to suppress or enhance specific types of signal. The types of lesions under study and the interval from injury to imaging impact on the sensitivity of a given neuroimaging sequence to such sequelae. The newer MRIbased techniques have yet to be systematically studied in mild TBI, and the link between demonstrable abnormalities, neurobehavioral deficits, and outcome in mild TBI remains to be determined (see McAllister et al., in press [36]). However, functional imaging techniques such as PET, SPECT, and fMRI show promise as tools to clarify the underlying pathophysiology of the sequelae of mild TBI. To date, most studies have focused on subjects with persistent neurobehavioral complaints, often a long time after injury, making it difficult to generalize the findings to the majority of patients with mild TBI. More work is needed in consecutive, unselected mild TBI populations followed over time to further clarify the role that these techniques may play.

Several studies have explored the utility of SPECT in TBI [37-41]. Many of these series consist of subjects with moderate, severe, or mixed injury severity, although some have included many subjects with mild TBI $[39,42]$. Most studies conclude that abnormalities in cortical perfusion can be shown even in the absence of structural abnormalities, and flow deficits observed with SPECT more accurately reflect the size or extent of damaged tissue than CT $[43,44]$.

Most PET studies in TBI have been conducted in moderate and severe TBI patients [45-49], although Humayun et al. [50] and Ruff et al. [51,52] demonstrated frontal and frontotemporal hypometabolism in mild TBI subjects with normal CT and MRI scans. Similar findings are reported by Gross et al. [53] in a retrospective series of 20 patients in treatment for postconcussive symptoms following a mild TBI, in whom abnormal metabolic activity, most commonly in temporal areas, was associated with post-concussive complaints and abnormal cognitive functions. While these studies suffer some methodological limitations, including small sample size and possible inclusion of patients with psychiatric complications capable of producing similar findings, they suggest that PET and SPECT may be more sensitive than MRI and CT scans in demonstrating brain dysfunction after mild TBI. 
Functional MRI (fMRI) also may also yield information regarding the nature of some postconcussive symptoms. Two reports [54,55] of individuals with mild TBI studied within one month of their injury showed different patterns of activation of working memory circuitry. Although cognitive performance was not different from that of healthy controls, the group with mild TBI reported significantly more cognitive and memory complaints. These findings suggests the possibility that the mild TBI group may have problems with the allocation of memory processing resources, and if this resource allocation difficulty is subjectively experienced, individuals may label this, for lack of a better term, as "memory trouble".

Several studies from populations of mixed injury severity (though they include many individuals with mild TBI) suggest that proton magnetic resonance spectroscopy (MRS) may reveal abnormalities not visible with conventional neuroimaging techniques, even in areas of "normal appearing white matter" [56,57]. Brooks et al. [58] demonstrated similar findings and found a relationship between imaging abnormalities and neuropsychological performance in the late period following TBI. Garnett et al. [59] also demonstrated altered MRS indices of cerebral metabolism suggestive of either a loss of normal cellular homeostasis or a relative alteration in normal cellular populations (increased glial cell density), in recently injured TBI subjects.

These findings offer clear evidence that advanced neuroimaging methods can reveal physiologically important abnormalities in patients with mild brain injury and some kinds of postconcussive symptoms. Importantly, many of these patients do not demonstrate abnormalities on conventional structural imaging techniques. As such, the presence of a normal CT or MRI scan cannot be equated with unequivocal absence of brain injury. Indeed, these findings alone suggest that newer neuroimaging methods may require reconsideration and revision of the traditional thesis that mild TBI is not typically associated with neuroimaging abnormalities.

\section{Electrophysiological measures}

A variety of electrophysiological techniques have been used to study brain function after mild TBI (for review see [60]). These techniques can be usefully grouped into four broad categories; standard electroencephalography (EEG), computerized or quantitative EEG (QEEG), evoked potentials (EP) (usually employ- ing an auditory or visual stimulus), and event-related potentials (ERP). EEG and QEEG are generally used to record the spontaneous electrical activity of the brain. EP and ERP studies typically measure brain activity in response to specific stimuli (for example an auditory "click"), and allow for repetitive measures and averaging of the stimulus-induced responses. Specific components of the stimulus-induced responses reflect processing of that stimulus by different brain regions (e.g., brain stem vs. cortex), and other characteristics of the waveforms induced by the stimulus (e.g., latency between peaks, or wave amplitude) can be used to infer characteristics of information processing in a given individual or population.

Schoenhuber and Gentilini [61] suggested that about $10 \%$ of patients with mild brain injury have persistent abnormalities when studied with standard EEGs, although this opinion is not universally shared [60, 62]. When present, conventional EEG abnormalities are typically nonspecific, such as mild disorganization of the background rhythms or a mild excess of slow wave frequencies.

Topographic brain electrical activity mapping and QEEG can demonstrate abnormalities not shown on routine EEG or evoked potential studies, although this is controversial [63]. Thatcher et al. [64] studied measures of EEG power spectral analyses in 608 patients with mild brain injury (GCS $=13-15$ and LOC $<$ 20 minutes). Their analysis permitted development of a discriminant function that separated mild brain injury patients from age-matched controls with remarkable accuracy. The location of the EEG abnormalities (frontal and frontotemporal, as well as changes in anterior-posterior patterns) was consistent with that typically associated with traumatic injury to the brain. This group has subsequently demonstrated correlations between certain QEEG measures, including coherence (a measure of the correlation of measured electrical activity between two different electrodes) and amplitude within different wave frequencies (i.e., alpha, beta, delta and theta), and MRI T2 relaxation times [65, 66]. Thatcher et al. [67] subsequently examined similar QEEG measures in mild, moderate and severe TBI, the results of which suggest a continuum of electrophysiologic abnormalities strongly related to the continuum of injury severity. These reports suggest that QEEG techniques may prove to be more valuable in the assessment of mild brain injury than standard EEGs, although their application in routine clinical practice for this purpose remains a matter of controversy [60].

A similar picture emerges with respect to the EP and ERP literature. Several studies have reported abnormal 
interpeak latencies using evoked potential paradigms in MTBI subjects [68-70], although these studies have not always been performed with the intent of correlating such findings with the presence or absence of specific postconcussive symptoms. Several studies have explored different EP paradigms indexing subcortical and thalamocortical processing [71,72] and visual evoked responses [73-76]. In general, some individuals with mild TBI have abnormal findings and it appears that the most robust correlations between specific EPs and clinical symptoms is found when the investigation targets dysfunction of the neural systems indexed by both.

For example, Arciniegas et al. [77,78] studied attentional gating mechanisms in individuals with persistent attentional complaints after TBI using a P50 evoked response to paired auditory stimuli paradigm. In a highly symptomatic and selected sample, individuals with persistent symptoms of impaired auditory gating demonstrated significantly abnormal P50 physiology. The authors suggest that this symptom and electrophysiologic abnormality are predicated on hippocampal cholinergic dysfunction. In a subsequent study, TBI subjects with these symptoms and abnormal P50 physiology were found to have smaller hippocampal volumes [79] and in an open-label study nearly $80 \%$ of these symptomatically and electrophysiologically improved during treatment with donepezil $\mathrm{HCl} 5 \mathrm{mg}$ daily. These findings are consistent with the hypothesis that hippocampal cholinergic deficits may underlie some of the attentional complaints in this group [80] and offer some support for the merits of using EP and ERPs to investigate the neurobiology of specific postconcussive symptoms.

It seems, then, that it is prudent to examine the consequences of mild TBI with respect to the specific postconcussive symptoms with which a patient presents. There appears to be reasonable support for this approach to evaluation, the goal of which is to understand such symptoms in terms of their individual neurobiologic underpinnings. While so doing, it is important to also be mindful of the symptomatic and etiologic overlap with other neuropsychiatric problems. With that in mind, we briefly review several of the common psychiatric complications of mild TBI to more clearly articulate this approach to the treatment of postconcussive symptoms.

\section{Postconcussive symptoms}

The most common "postconcussive" symptoms encountered after a TBI can be grouped into three categories: cognitive complaints (decreased memory, attention, and concentration), somatic complaints (headache, fatigue, insomnia, dizziness, tinnitus, sensitivity to noise or light), and affective complaints (depression, irritability, and anxiety). In the immediate post-injury period $80-100 \%$ of mild brain injury patients will describe one or more symptoms reasonably attributable to their injury [81]. Mirroring their subjective complaints, individuals with mild brain injury show deficits in speed of information processing, selected tests of attention and memory, and performance consistency in the first week or so subsequent to injury [81-86]. Studies of cognitive testing at 1 month and 3 months subsequent to injury tend to show progressive diminution of such deficits, although those that persist are usually in these same three areas of cognitive function [83,85-88]. Individuals whose initial injuries include complications such as depressed skull fractures, contusions, and subdural or epidural hematomas may be more likely to have persistent deficits in speed of information processing, verbal and recognition memory, and verbal fluency [26]. Fortunately, the majority of patients experience complete resolution of these symptoms even if only after some delay.

Several studies demonstrate high rates of symptoms even three months after injury. Rimel et al. [89] studied 424 patients with mild brain injury (GCS $>13$, LOC $<20$ minutes) and found that $78 \%$ of their patients complained of headache; $60 \%$ complained of decreased memory; and $50 \%$ and $25 \%$ complained of either decrease in financial status or were unemployed respectively 3 months after their injury. Levin et al. [81] reported $47 \%, 22 \%$, and $22 \%$ of the mild TBI patients continued to complain of headache, decreased energy, and dizziness, respectively, at three months. Bohnen et al. [88] report that three months after injury 54\% remained symptomatic to some degree, and $25 \%$ of the sample had three or more symptoms, headache, fatigue, dizziness, and concentration being the most common symptoms. Ingebrigsten et al. [90] reported $62 \%$ with one or more symptoms and $40 \%$ with 3 or more symptoms 3 months after mild TBI (GCS $=13-15$, LOC, no focal neurological deficits or abnormal CT findings). Unfortunately, most studies have not determined or reported base rates of postconcussive symptoms for healthy controls.

Postconcussive symptoms may persist even six months after injury. Bohnen et al. [88] reported that almost $25 \%$ of their sample had three or more symptoms at six months, and at both 3 and 6 months, the group with 3 or more symptoms showed reduced performance 
on a measure of complex attention and reduced tolerance to light and sound compared to healthy controls. A recent study by McCullagh et al. [35] found significant rates of persistent symptoms 5-6 months after mild TBI with $50 \%$ of the 57 subjects reporting dizziness and headache, and $\sim 75 \%$ reporting fatigue. Furthermore, $50-60 \%$ of those with GCS scores of 13-15 met General Health Questionnaire [91] criteria for psychiatric "caseness" indicative of significant psychological distress.

Even after six months, several studies have suggested that a substantial number of patients continue to experience postconcussive symptoms after mild TBI, although the frequency of such problems and the characteristics of those experiencing them is a matter of substantial controversy. Deb and Koutzoukis [18] evaluated 140 individuals (134 face to face interviews) who had sustained mild TBI one year earlier and found almost 30-33\% had either moderate or severe disability on two different outcome measures and $55 \%$ had at least one ongoing postconcussional complaint. Unfortunately there was no control group that would allow for comparison of base rates of the target symptoms in the general population.

If one limits the inquiry to those with uncomplicated mild TBI, outcomes appear to be more favorable. Alves et al. [92] followed 587 consecutive admissions for mild TBI (GCS $=13-15$, normal skull films and CT scans, hospitalization $<48$ hours) and found that $10-40 \%$ were symptomatic one year after injury, headache being the most common complaint. Importantly, relatively few (2-5\%) suffered multiple complaints suggestive of a postconcussive syndrome. It is possible that low rates of persistent symptoms reflect the effect of education at the time of injury, but the lack of a comparison group in this study leaves uncertain this strength of effect of such interventions regardless of their form.

In terms of more objective measures of longer-term cognitive deficits, the situation is a bit murkier. In two carefully performed studies Dikmen et al. were unable to find significant differences between an unselected TBI group (i.e., not specifically complaining of cognitive symptoms), and a non-injury comparison group [93] or between a TBI group and an other-injury comparison group $[9,10]$ one year after injury. Binder et al. [94], in a meta-analysis of data from eight studies of long term effects of mild TBI, found fairly small effect sizes on measures of attention, but in a review of additional studies reported that approximately $8 \%$ of individuals remained symptomatic chronically and that $14 \%$ had work-related disability [95]. However in a group of 53 symptomatic patients with mild brain injury (GCS $>13$, LOC $<20$ minutes) examined 6 to 8 months after the injury, Leininger et al. [96] found significant impairment on four of eight neuropsychological tests assessing information processing, reasoning, and verbal learning. Guilmette and Rasile [97] demonstrated significant deficits in tests of verbal memory and learning in a sample of individuals with mild TBI (LOC $<30$ minutes, PTA $<24$ hours) and persistent cognitive complaints, suggesting that the persistently symptomatic group may have very different characteristics from an otherwise unselected group of mild TBI subjects. As such, it seems that any estimate or consideration of persistent neuropsychological dysfunction following mild TBI should account not only for the severity of TBI but also more specifically for the presence of cognitive complaints.

Regardless of the exact percentage of individuals that are symptomatic in the late post-injury period, there is an unquestionable discrepancy between the message typically given to the individual with a mild TBI in the Emergency Department ("You had a very mild injury or concussion, you will be fine ...") and the reality that many of these individuals experience. This suggests, at a minimum, that the traditional wisdom of uniform and full recovery following mild TBI requires reconsideration.

At least some individuals report subjective distress that appears disproportionate to the initial severity of the injury experienced. While it is certainly possible that there is a reasonable neurobiological explanation for this would-be disparity between initial injury severity and injury outcome, such observations have prompted investigation of the role of motivational factors (and particularly compensation) in the genesis of postconcussive symptoms. At the outset of this discussion, it is important to note that many of the investigations addressing this issue are performed in medicolegal practices or at least recruit subjects for study from such practices; therefore, these studies are based on a highly selected population that may not be representative of the overall MTBI population (e.g. [1]).

Binder et al. [2] exploring the relationship between litigation and symptoms after TBI published a metaanalysis of some 18 studies including 2,353 individuals with TBI of varying severity and found a weighted mean effect size of 0.47 ; based on this data, they suggest that financial incentives may account for $20-25 \%$ of the persistent signs and symptoms associated with TBI. Feinstein et al. [4] prospectively studied the role of litigation on symptoms in 97 consecutive individu- 
als with mild TBI seen about six weeks after injury. Even this early in the process, those involved in litigation were experiencing significantly more anxiety and social dysfunction, and had poorer outcomes on the Glasgow Outcome Scale [98] and the Rivermead Head Injury Follow-up Questionnaire [99] than nonlitigants. Other studies fail to find significant associations between compensation or litigation, and frequency or severity of postconcussive symptoms [89, 100-102]. Given the prolonged and highly adversarial nature of many litigation and compensation proceedings, it would be surprising if the kind of psychosocial stress associated with the litigation process did not adversely affect psychosocial functioning and symptom presentation.

Other motivational factors may also play a role in functional level and cognitive performance. Keller et al. [3] compared performance on a test of divided attention in 12 individuals with MTBI, 10 with more severe injuries, and 11 healthy controls before and after being told that performance might impact on ability to drive safely. The MTBI group did significantly better, and in fact performance was within published normal range with driving as a motivator. However, the healthy controls also improved and still outperformed the MTBI group.

A variety of tests have been developed to help with the assessment of individuals in whom motivation and malingering are being questioned (see Iverson and Binder [5] for discussion). Many of these tests are based on a forced choice format in which performances significantly worse than chance, or in some cases scores lower than norms from populations with severe neurological disorders, are interpreted as suggesting a negative response bias $[5,103]$. Such tests cannot, in isolation, offer a reliable measure of motivation or response bias. It is important to assess consistency of performance over several tests that assess several cognitive domains such as memory, attention, and learning, rather than staking an opinion on a single test. Inconsistencies in performance must also be interpreted within the context of potential fatigue, medication effects, and medical or co-morbid psychiatric conditions; without consideration of such confounding factors, it is not appropriate to assume that any performance deficits indicate malingering. Like the non-injured population, individuals with MTBI are subject to the influence of stress and the same complex motivations that influence behavior in all individuals. Performance variation under various different conditions, or worsening of symptoms in the context of heightened stress such as ad- versarial litigation is "normal" and should not be interpreted as unequivocal evidence of malingering, or that "real injury" is not present.

It seems reasonable to conclude that most patients experiencing a mild TBI endorse postconcussive symptoms within the immediate post-injury period. Significant resolution of these symptoms occurs in about half of the patients by 1 month and roughly two-thirds at 3 months. Several authors have suggested that "organic factors" cause initial postconcussive symptoms and psychological "issues" drive the maintenance and elaboration of persistent symptoms [104-108]. However, King et al. $[109,110]$ found that depression, anxiety, and injury impact correlate highly with initial symptoms, and account for $53 \%$ and $23 \%$ of the variance in postconcussive symptoms at three and six months after injury respectively, suggesting that psychological factors play a role in symptoms formation even before the development of "persistent" symptoms. Although preinjury vulnerabilities such as personality style, prior injuries, age at injury, and psychosocial support system may impact outcome after mild TBI (see Kay [111] for a more complete discussion), it does not seem reasonable to attribute all persistent postconcussive symptoms to such factors alone. As noted earlier, many reports describe persistent electrophysiologic and neuroimaging abnormalities in mild TBI patients with persistent postconcussive symptoms, suggesting that neurobiological factors also contribute to persistent symptoms. The more simplistic temporal dichotomization of symptom formation and persistence into "organic" and psychological factors, respectively, appears to be misleadingly oversimplified, if not frankly inaccurate.

\section{The postconcussive syndrome}

The term postconcussive syndrome is used inconsistently in the literature and in clinical settings, with its referents including both postconcussive symptoms and also a multitude of other comorbid and/or complicating medical and neuropsychiatric problems. However, it is not clear that there is in fact a postconcussive "syndrome". Indicators of a syndrome include consistent symptom linkage (i.e., if symptom " $\mathrm{A}$ " is present, there is a high likelihood that symptom " $\mathrm{B}$ " will be present), and coupling of symptom response to treatment (i.e., if symptom "A" is improved by treatment " $\mathrm{X}$ ", there is a high likelihood that symptom "B" will also respond to a similar degree to treatment "X"). Several studies report that headache, dizziness, and fatigue or 
decreased energy are the most common postconcussive complaints [35,81,88,92]. Several studies indicate that it is common to have symptoms in more than one area (e.g., headache, fatigue, memory problems), especially early in the recovery period [81,92], with smaller percentages maintaining multiple symptoms over time. Some of these studies suggest that those with multiple symptoms at three months post-TBI are those most likely to continue experiencing multiple postconcussive symptoms at 6 and 12 months. While consistent types of complaints across these studies might support the validity of a postconcussive syndrome, these symptoms have high base rates in the general population and are somewhat non-specific in nature [6,8-10,87].

Even when these symptoms are reasonably attributed to the effects of mild TBI, it may still merely be the case that the persistence of multiple symptoms at three months and later simply reflects the effects of relatively more severe injuries in the mild TBI population. Among these patients, the studies above suggest that relatively few individuals experience persistence of their entire set of multiple symptoms over time, and instead maintain only some subset of those symptoms. This uncoupling of postconcussive symptoms from one another (some get better while others persist) argues against the concept of a postconcussive syndrome.

It may therefore be more accurate to assert that common symptoms arise as a result of injury to brain areas commonly affected by TBI, and that these symptoms occur to greater or lesser extent as a function of the particulars of an individual injury and relevant premorbid factors. In this context, it may be most useful to identify specific symptom patterns and to regard such patterns as reflective of the most injured areas of brain in a given individual. For example, the type, location, and severity of injury in a patient experiencing mild intermittent headache and dizziness several months after a mild TBI may be dramatically different than that in a patient experiencing severe memory impairments, fatigue, chronic pain, and balance problems 2 years post-MTBI.

As such, conceptualizing the sequelae of TBI or mild TBI as a syndrome (in the strict sense of the word) entails a consistency of clinical presentation that is not readily supported by the clinical studies of this population. Individuals with multiple symptoms in the immediate post-injury period may experience improvement in all, some, or none of the symptoms over time, suggesting at the very least that the symptoms are not always tightly linked and can be uncoupled. In this light, the concept of a postconcussive syndrome seems difficult to support. Maintaining this concept may negatively influence approach to treatment. If one considers the multiple postconcussive symptoms as those of a syndrome, and therefore regards them as products of a common underlying mechanism (be it neural damage, depression, or malingering), one tends to look for treatments directed at that single underlying problem in order to ameliorate the syndrome as a whole. Neither common clinical experience nor the litany of medication and other clinical trials performed to-date (see Arciniegas et al. [112] for a review) support this approach to treatment; multiple and varied treatments are generally required for the multiple and varied symptoms of these patients. If one instead views these symptoms as the products of several different injury locations and neuropathologies relevant to injury at those locations, then one tends to take a more careful look at the typology of each symptom and to properly diagnose and treat these different sources of distress and disability. As is the case with any other medical condition, this conceptualization of persistent symptoms would in no way exclude modulation of symptom presentation and symptom intensity by other factors including depression, pain, and secondary gain.

The more we learn about the etiology of the different symptoms commonly seen after TBI, the more clear it is that specific symptoms have specific underlying mechanisms and by extension each requires specific treatments. Efforts are ongoing to resolve these controversies by investigating the neurobiology of TBI and postconcussive symptoms. The development of rational evaluation methods and treatment approaches requires clarification of the above issues, and must translate recent advances in the neuroscience of cognition, emotion, and behavior into a set of tools for the treatment of commonly occurring problems after TBI.

\section{Psychiatric complications}

In addition to the cognitive sequelae described above, a variety of significant emotional and behavioral sequelae are associated with mild brain injury, many of which suggest that TBI confers an increased vulnerability to psychiatric disorders during and subsequent to the acute recovery period [113]. Depressive syndromes, anxiety, post-traumatic stress disorder (PTSD), sleep disturbances, and somatization are particularly relevant to the discussion of postconcussive symptoms as they are common complications of mild TBI, may amplify the experience and reporting of postconcussive symptoms, and be a source of excess distress and disability. 


\subsection{Depression}

Depressive symptoms are a very common complication of mild brain injury (see [114] for review). Gfeller et al. [115] found a relationship between depression, elevated postconcussive symptoms, and impaired performance on some cognitive measures in their sample of 42 individuals with mild TBI and headache. McAllister and Flashman reported a similar overlap in a sample of individuals with mild TBI referred for cognitive evaluation [116]. Studies of emotional distress following various initial severities of TBI also suggest that depressive symptoms are elevated in this population $[15,117,118]$. Fann et al. [119] described a frequency of depression after mild TBI of $26 \%$, similar to the results reported by Federoff et al. [120] and Jorge et al. [121,122] in which about $25-30 \%$ of the group was depressed one month after their TBI and at about one year post-TBI. Schoenhuber and Gentilini [123] reported significantly higher rates of depressive and anxiety symptoms among patients approximately 9 months following MTBI than in a group of matched comparison subjects drawn from friends and relatives. Mobayed and Dinan [124] reported that $30 \%$ of their 55 mild brain injury patients demonstrated evidence of an affective disorder on the Leeds scale [125], with $20 \%$ meeting DSM-III criteria for major depression.

Although depression is a common consequence of TBI in general, many postconcussive symptoms such as subjective slowing, irritability, fatigue, and sleep disturbance may misleadingly suggest an active depressive syndrome, even when patients do not endorse explicitly items such as "depressed mood". Saran [126] reported that depression following TBI differs from that of noninjured depressed patients, in that depressed TBI patients manifest less diurnal variation, less anorexia or weight loss, and less psychomotor retardation or agitation. It is also important to evaluate and treat depression aggressively in light of the observation that it adversely affects TBI outcome [127]. Thus depressive symptoms are a common complication of a mild TBI, with major depression occurring in between $20-30 \%$ of those with complicated mild injuries. Depressive symptoms can be a significant contributor to psychiatric disability subsequent to mild brain injury either as a component of many postconcussive symptoms, or as a discrete major depressive episode. Patients with a prior history or family history of depression may be at greater risk to develop depressive symptoms subsequent to injury although the majority of depressive episodes arise in patients with no such vulnerabilities.

\subsection{Anxiety and posttraumatic stress disorder}

Few studies have examined anxiety syndromes after mild brain injury. As with "depressive" symptoms, many postconcussive symptoms and core symptoms of generalized anxiety disorder are similar. Many patients endorse complaints of headache, dizziness, blurred vision, irritability, and sensitivity to noise or light after mild brain injury $[81,87,93,128]$. It is less clear how many patients actually experience anxiety and how many have diagnosable anxiety disorders. Although $55 \%$ of subjects in a study by Dikmen et al. [87] complained of subjective anxiety, $45 \%$ of the matched controls had similar complaints (a statistically nonsignificant difference). Schoenhuber and Gentilini [123] did not find a significant difference in mean anxiety scores in their study of 35 mild brain injury patients and matched controls. In the study by Fann et al. [119], $24 \%$ of their sample (the majority of whom had mild TBI) met criteria for generalized anxiety disorder when evaluated 2-3 years after TBI.

There is an increasing awareness of the relationship between posttraumatic stress disorder (PTSD) and brain injury. It is not uncommon in clinical practice to see patients with a history of mild brain injury who demonstrate signs and report symptoms suggestive of PTSD. These include sleep disturbance, recurrent nightmares, exaggerated startle responses, daytime flashbacks, and avoidant behaviors such as refusing to drive or leave home. Interestingly, Mayou et al. [129] report that $48 \%$ of those with a mild TBI with definite LOC had PTSD 3 months after injury, and a third of subjects with any mild TBI had PTSD one year after injury. Although it might at first seem strange that those with loss of consciousness could develop PTSD with intrusive memories (given that LOC would preclude awareness during the TBI-inducing event), it has been suggested that the intrusive memories are of events immediately prior to or after the accident, or there may be patchy amnesia with some islands of preserved memory.

Bryant and Harvey report that rates of Acute Stress Disorder (ASD) one month after motor vehicle accidents are comparable among patients with and without TBI from such accidents, and that ASD predicts the development of PTSD six months after injury [130-135]. The rates of PTSD are surprisingly high: $20 \%$ in the TBI group and $25 \%$ in the non-TBI group. Importantly, the TBI group endorsed more postconcussive symptoms than did the non-TBI group despite their shared psychiatric diagnosis. This suggests that PTSD may 
amplify postconcussive symptoms after a mild TBI and thereby complicate recovery.

Anxiety symptoms and disorders, including PTSD, are relatively common among patients with mild TBI and overlap substantially with postconcussive symptoms. Attributing such symptoms to premorbid conditions, the direct effects of neuronal injury, or secondary reactions to trauma and events in the peri-injury period is very challenging. Nonetheless, evaluation of such symptoms from at least these perspectives is a requisite part of the evaluation of the patient presenting with such symptoms after TBI.

\subsection{Sleep disturbance}

Patients may develop a variety of sleep disturbances following mild TBI [136], with the frequency of sleep disturbances following TBI ranging from $36 \%$ to $70 \%$ [137]. Sleep disturbances may include disorders of initiating and maintaining sleep, disorders of excessive daytime somnolence, and less commonly parasomnias. These disturbances may be a direct consequence of injury to reticular-diencephalic systems participating in the regulation of sleep-wake cycles and circadian rhythms, a manifestation of a comorbid psychiatric disorder or consequence of mild TBI, a consequence of pain, may result from treatments of other TBI-related symptoms, or may result from some combination of these and other factors. Sleep disturbances following TBI are commonly associated with cognitive, emotional, and behavioral disturbances and may significantly affect the course of recovery from TBI. Despite the frequency of this problem, the prevalence, clinical features, types of sleep problems, relationship between sleep disorders and other psychiatric problems, and treatment of these conditions remain an understudied set of questions. Nonetheless, TBI related sleep disorders may amplify or even mimic other postconcussive symptoms (i.e., cognition, fatigue, irritability) and also mood or anxiety disorders. The commonly applied treatments for sleep disturbances (e.g., benzodiazepines and other sedative-hypnotics) may mimic or exacerbate postconcussive symptoms, and particularly cognition and fatigue, as well. Conversely, depression, anxiety, pain, seizure disorders, and other postconcussive problems as well as treatments for these problems may interfere with sleep. Complaints of sleep disturbance in these patients require consideration of these several possible and interacting etiologies prior to initiating any treatment.

\subsection{Somatization}

There is a small group of individuals (though they can make up a large percentage of certain referral practices) who have multiple disabling cognitive, affective, and somatic complaints suggesting a cause other than uncomplicated MTBI alone. These symptoms may develop well after the injury, typically progress in severity rather than improve with time, and the subjective distress is often of a much greater degree than the functional level of the individual would imply or that would be expected given the severity of the injury. Neurodiagnostic tests, both conventional and experimental (QEEG, PET, SPECT, etc.), are frequently within normal limits. The history often suggests an exquisite sensitivity to everyday physiological sensations and events. In many respects, these patients appear to have an acquired somatoform disorder, although the life-long history of somatizing behavior needed to make this diagnosis is usually absent. Nonetheless, these patients can be as difficult to treat as the lifelong somatoform patient.

Although the contribution of compensation to the clinical picture should be considered, it may be that TBI increases the risk for somatoform disorder just as it does for depression, anxiety, and PTSD. Furthermore, in the absence of a pre-morbid history of similar symptoms, it is hard to escape the conclusion that their disability results from the injury, even if not from damage to neural tissue. The mechanisms by which this problem develops after TBI are not established, and therefore it is not reasonable to conclude that this problem represents a simple psychological reaction; while this may be the case, research addressing the possible neurobiological and psychosocial factors involved in the genesis of post-TBI somatization is required before any interpretations may be made.

\section{Approach to evaluation and treatment}

At the risk of stating the obvious, the foundation of the approach to treatment of patients with mild brain injury is a proper evaluation. Significant effort must be expended to clarify premorbid history. In particular one must look for a prior history of brain injury, which can be seen in as many as $30 \%$ of patients [89]. The association of substance abuse with brain injury is well described [138] and may contribute to post-injury sequelae. Interviews with significant others can be invaluable in gaining a clearer picture of these issues. 
Without a clear picture of premorbid functional level it is impossible to determine change in function after the injury.

Signs and symptoms must be clearly defined, as well as changes in symptom picture as a function of time from the injury. The profile of the injury itself must be outlined, including the type of injury, the presence or absence of loss of consciousness and its duration, and the presence, absence, and duration of any retrograde and anterograde amnesia. Corroborative information including accounts from observers, emergency medical technicians, ambulance and emergency room personnel, and inpatient hospital records can be invaluable. When evaluating these records, phrases such as "normal mental status" without sufficient documentation do not eliminate the possibility that there were cognitive changes. This is particularly true when the emergency team is distracted by other trauma such as injury to the spinal cord [139]. The presence or absence and location of complications such as depressed skull fractures, cerebral contusions, and extradural hematomas should be noted because of the potential prognostic complications [26]. The neurodiagnostic tests done and the timing in relation to the injury should be clarified, and the reports or actual studies obtained. All of the above information can then be integrated with findings from the clinical interview to determine the consistency of the history and exam with the known sequelae of mild brain injury.

This process should determine whether the clinical presentation is consistent with what is known about the usual sequelae and time course of recovery after mild TBI. Certain features of the history and presentation should raise concerns that other factors are at work. Subjective report and objective function should be at least reasonably consistent with one another. A functional level that is grossly inconsistent with the complaints presented (e.g. individual reports severe disabling memory loss yet gives a clear and detailed history, and drives self to appointment without assistance) is worrisome for the presence of etiologic factors other than TBI-induced neurobiological dysfunction. The time course of the symptoms is important to attend to. Symptoms which did not start shortly after the injury, or which have gotten worse over the subsequent months or years are not likely due simply to an uncomplicated mild TBI. Performance on standardized neuropsychological testing that is dramatically worse than functional level is concerning for the variety of reasons described earlier. The presence of any of these features should encourage an aggressive search for co-morbid, or confounding conditions.
A very careful assessment should be done for depression, anxiety disorders, PTSD, and sleep disturbances. It is important to be aware that these disorders may present atypically in the context of brain injury. Although somatization and malingering are possibilities in this context, there are relatively uncommon, if not frankly rare, conditions even in medico-legal settings and should not be the default diagnosis when atypical features are noted.

Treatment should then follow rationally from this diagnostic scheme. The finding of PTSD, anxiety, or depression should prompt an aggressive treatment plan that includes appropriate psychotherapeutic and pharmacologic interventions. Brief cognitive behavioral therapy has been suggested to be of particular benefit in the treatment of postconcussive symptoms, and may limit the potential impact of iatrogenic development of persistent cognitive and emotional symptoms following mild TBI [140]. Selection of pharmacologic agents is undertaken with consideration of both literature offering evidence of benefit and also the particularly untoward effects of some agents in the TBI population. Whether these co-morbid psychiatric, neurological (e.g., headache, seizure), or other-injury related conditions are present or not, one should not assume that all symptoms are accounted for by such associated illnesses. Rather, aggressive assessment of specific postconcussive symptoms, be they cognitive, emotional, behavioral, or somatic, should be undertaken and appropriate interventions dictated by the findings pursued.

\subsection{Education}

Often the most effective intervention in patients with active neurobehavioral sequelae is a careful explanation of the pathophysiology, typical sequelae, the usual time course of recovery associated with mild brain injury, and also the potential for longer-term difficulties [141147]. Such interventions appear most effective when offered soon after the injury and in the presence of family, friends, or significant others (see Wrightson [148]). Early intervention may avoid the psychological, social, occupational, and medicolegal complications attendant to the patient and others questioning the validity of complaints based on the seemingly "mild" nature of the injury and the patient's healthy appearance. Careful validation of the complaints of the patient without undue fostering of illness behavior is appropriate, and should be coupled with setting individualized and realistic goals for return to major activities and employ- 
ment. It is usually best to do this kind of goal-setting with the involvement of key stakeholders (patient, significant others, employers, payors, etc.), rather than in a prescriptive, one approach-fits-all manner.

\section{Medication approaches}

\subsection{General principles}

Most authors suggest following several general principles of pharmacologic management in this population. Indications for all active prescriptions should be reviewed, and all non-essential medications should be discontinued. Inquiring about the patient's use of over-the-counter (OTC), herbal, and other supplemental agents is important in light of their common use, potential adverse effects on cognition (particularly OTC "sleeping pills" containing scopolamine), and significant potential for interaction with prescribed medications. Target symptoms should be assessed and reassessed during treatment using standardized assessment tools, including postconcussive symptom rating scales, standard psychiatric inventories, neurobehavioral measures, or neuropsychological performance. Because individuals with MTBI seem to be more sensitive to common medication side effects such as sedation, psychomotor slowing, and cognitive impaired, it is prudent to use lower starting and (often) final doses, and spread out the titration intervals [112,149-153]. Careful monitoring of treatment effects, side effects, and drug-drug interactions is particularly important in these patients.

Medication approaches to the sequelae of mild TBI generally take three broad approaches: amelioration of psychiatric complications, amelioration of specific somatic symptoms (e.g., headache, dizziness, sleep disturbances) and augmentation of cognition. At present, there are no Federal Drug Administration (FDA) approved treatments for cognitive, emotional, or behavioral impairment due to TBI; hence, pharmacotherapies are generally modeled after those for patients with phenomenologically similar but etiologically distinct disorders (i.e., attention-deficit hyperactivity disorder, Alzheimer's disease, etc.). For example, the attention deficits of persistently impaired TBI survivors sometimes superficially resemble those of patients with attention-deficit hyperactivity disorder (ADHD) prompting treatment with psychostimulants, and primary major depressive episodes are sufficiently comparable to those following TBI to suggest potential benefits of antidepressant therapies.

\subsection{Approach to psychiatric complications}

With respect to amelioration of psychiatric complications, the same general approaches taken in the noninjured population are typically used, although there are very few reports describing good clinical studies of these agents in this population (see Arciniegas et al. [112] for additional review and specific recommendations). Although a now remote study by Saran [126] suggests that some of these patients may be less responsive to tricyclic antidepressants than patients without a brain injury, a recent study by Fann et al. [154] reports improvement in depression after TBI during treatment with sertraline. Our experience is that most serotonergically active antidepressants are effective for the treatment of depression after TBI and reasonably useful for the treatment of anxiety disorders after TBI, but that these patients often require smaller starting doses and cautious dose-escalation. The phenomenology of depressive and manic syndromes can also be altered by a brain injury [126,151,155-157], resulting in a mixed and atypical clinical presentation. While the optimal treatments for such problems are not clear at present, anticonvulsant mood stabilizers appear useful when secondary manic symptoms, irritability, and/or aggression are features of the clinical presentation. Thus psychotropic use is complicated by enhanced sensitivity to side effects, a mixed and atypical clinical picture (which can complicate assessment of target symptoms and drug response), and perhaps a reduced efficacy of certain standard agents, although the evidence for this is tentative.

\subsection{Approach to treatment of specific somatic symptoms}

Postconcussive somatic symptoms such as headache, dizziness, pain, seizures, visual disturbance, and hyperacusis must be evaluated according to the general principles described above. Detailed discussion of the treatments for each of these problems is well beyond the scope of the present work, but a few additional principles may be useful to consider. Many of these problems require provision of care by providers from multiple healthcare disciplines; effective communication is essential to delivering a coordinated and effective set of nonpharmacologic and pharmacologic treatments. Where possible and appropriate, non-pharmacologic interventions should be applied in the service of promoting functional recovery. For example, this includes teaching and reinforcement of sleep hygiene, a 
brief course of neuro-optometric rehabilitation, physical therapy and related interventions for pain, relaxation training for posttraumatic headaches, and so on. When pharmacologic interventions are necessary, it is prudent to select agents whose effects may address multiple target symptoms. For example, a patient with irritability, sleep disturbance, posttraumatic headaches, and chronic cervical pain might be well served by treatment with an anticonvulsant at night, the effects of which may confer some relief from each of these problems. Alternatively, a patient with depression, affective lability, and posttraumatic headache might benefit from treatment with either an SSRI or low-doses of a serotonergically potent tricyclic antidepressant.

\subsection{Approach to augmenting cognition after TBI}

The treatment of postconcussive cognitive symptoms also involves both nonpharmacologic and pharmacologic methods. Cicerone et al. [158] offer guidelines and recommendations for cognitive rehabilitation strategies based on a review of the treatment literature in this area. Although there are few randomized controlled trials of these treatments, there is evidence to support their use for the treatment of memory, attention, executive function, and communication deficits following TBI. These treatments appear to be most useful when offered to reasonably high-functioning and well-motivated patients.

The best use of pharmacologic therapies for cognitive impairments following TBI is less certain. The clinical research of the past two decades in this field suggests that catecholaminergic and cholinergic mechanisms are important in the genesis and augmentation of attention and memory after TBI. Catecholaminergic mechanisms, particularly those in DA and alpha2 adrenergic (A2A) systems, appear to play important roles in working memory (WM) function (see Arnsten, 1998 for review [159]) both in normal individuals and individuals with TBI. Improved spatial working memory performance has been shown in healthy individuals treated with both bromocriptine $[160,161]$ and methylphenidate [162]. McDowell et al. [163] found significant improvement in tasks requiring executive function (e.g. dual-task paradigm) but not WM storage capacity or prefrontal tasks which did not require executive functions following a single dose of $2.5 \mathrm{mg}$ bromocriptine in 24 TBI subjects. Several dopamine (DA) agonists including bromocriptine, amantadine, and psychostimulants such as methylphenidate, dextroamphetamine, and L-dopa have been used to treat cognitive and behavioral sequelae of TBI and other acquired brain injuries. Clinical observations document improvement in many subjects in areas as diverse as impulse control, motivation, attention, insight, anger/aggression, and memory $[112,150,164-$ 175]. These observations suggest that dopamine is important in the maintenance of arousal, attention, working memory, executive function, and other related aspects of cognition. However, there are no neuroimaging or neuropathological studies that demonstrate persistent dopaminergic dysfunction in the late period following TBI. Hence, the exact mechanisms by which these agents are useful in postconcussive patients with cognitive complaints remain uncertain.

A2A mechanisms also appear to play a prominent role in the activation and modulation of WM. Localized and global depletion of catecholamines (DA and $\mathrm{NE}$ ) as well as aging impair performance on spatial WM tasks, with effects similar to that seen with ablation of neural tissue in the prefrontal region [159,176180]. Infusion of A2A antagonists produces spatial WM impairment in both monkeys and rats [181,182] that can be reversed by administration of $\mathrm{A} 2 \mathrm{~A}$ agonists (see Arnsten [159] for review). A1A agonists can also impair WM function, suggesting that different adrenergic receptors have opposing effects on cognitive function [183] and that it is important to clarify the different roles of both these receptor families and the medications that affect them. Thus broad-spectrum adrenergic agents (e.g., methylphenidate) that augment the endogenous release of dopamine and norepinephrine may have mixed effects on WM function as a function of the multiple receptors and neurotransmitter systems they influence. More specific agents like guanfacine (an A2A agonist) can improve WM in healthy individuals $[184,185]$ and may improve working memory after TBI but additional ongoing research requires completion to test this hypothesis.

Another hypothesis relates cognitive impairment following TBI to acute and long-term alterations in cortical cholinergic function [77-80]. Animal studies [186188] demonstrate chronic alterations in hippocampal cholinergic function following experimentally-induced TBI and the relationship of such alterations to persistent cognitive impairments. Human post-mortem studies $[189,190]$ also demonstrate that TBI produces cortical cholinergic dysfunction via loss of cortical cholinergic afferents; these studies also demonstrate that postsynaptic muscarinic and nicotinic receptors are not reduced by TBI. This suggests that increasing levels of cortical acetylcholine might afford improvements in 
post-TBI cognitive impairments predicated on cholinergic dysfunction. Importantly, this hypothesis is complementary to the catecholamine hypothesis in that the role of acetylcholine is, in many parts of the cortex, that of a facilitator: acetylcholine is largely excitatory, and increased cortical levels appear to increase the likelihood of neuronal firing upon receipt of another excitatory neurotransmitter like DA or NE. In that context, one might improve cognitive dysfunction related to cholinergic deficit by either increasing levels of acetylcholine or by sufficiently increasing the levels of catecholamines such that the effects of cholinergic deficit are overcome in the areas whose function is dependent on both.

Multiple studies demonstrate that cholinergic augmentation, generally using one of several cholinesterase inhibitors (e.g., physostigmine, donepezil) can improve TBI-induced attention and memory deficits even in the late post-injury period ( $>1$ year) in some TBI survivors [191-198]. Arciniegas et al. have advanced the hypothesis that cholinergic mechanisms play a critical role in the genesis of certain attentional deficits following TBI $[77,78]$, deficits that appear robustly responsive to donepezil $\mathrm{HCl}$ [80].

Thus both theoretical and clinical evidence suggests that the cautious, empiric use of cholinergic and catecholaminergic agents is warranted for the treatment of chronic memory and attentional deficits. It may be that both cholinergic and dopaminergic dysfunction, including dopaminergic dysfunction secondary to reduced cortical cholinergic function, contribute to cognitive impairments following TBI. While the literature demonstrating reduced cortical cholinergic function following TBI is robust, clinical evidence suggests a role for augmentation of either or both neurotransmitter systems in persistently impaired TBI survivors. However, the inter-individual response to such agents is not uniform [199]. Some patients respond to psychostimulants, some to cholinesterase inhibitors, some to either and others to neither class of medication. Selecting an appropriate treatment for a given patient might be done more easily if clinical markers (neurophysiologic, neuroimaging, or specific symptoms) of dysfunction in these systems were available. However, no simple and inexpensive methods of indexing either dopaminergic or cholinergic function in humans are available presently. At present, we recommend the psychostimulants for patients with prominent attentional and/or working memory impairment, with or without comorbid hypoarousal, apathy, or depressed mood, and the cholinesterase inhibitors for patients in whom memory impairments and impaired sensory filtering (or "impaired sensory gating") are the predominant clinical problems.

\section{Conclusion}

The evaluation of postconcussive symptoms requires an understanding of the multiple factors relevant to the production and maintenance of symptoms following trauma to the brain. These factors include the neuropathological profile of the typical traumatic brain injury, the course of recovery, the susceptibility of course and recovery to various psychosocial factors, and the interplay between these factors and the enhanced vulnerability to de novo psychiatric disorders that brain injury confers on the individual. Treatment requires an accurate assessment of the primary driving factors involved in the genesis and maintenance of the symptoms, and understanding of the neurochemistry of prominent cognitive domains vulnerable to traumatic injury, and an individualized, flexible, multi-faceted treatment plan a neurobiopsychosocial approach.

\section{Acknowledgements}

This work was supported in part by a Research Career Development Award from the Department of Veterans Affairs Research Service (Dr. Arciniegas), and NIDRR Grants H133G70031 \& H133000136; NIH Grant RO1 NS40472-01, the Ira DeCamp Foundation, and New Hampshire Hospital (Dr. McAllister).

\section{References}

[1] H. Miller, Accident neurosis, British Medical Journal 1 (1961), 919-925, 992-998.

[2] L.M. Binder and M.L. Rohling, Money matters: a metaanalytic review of the effects of financial incentives on recovery after closed-head injury [see comments], American Journal of Psychiatry 153(1) (1996), 7-10.

[3] M. Keller et al., Reversible neuropsychological deficits after mild traumatic brain injury, Journal of Neurology, Neurosurgery \& Psychiatry 68(6) (2000), 761-764.

[4] A. Feinstein et al., The effects of litigation on symptom expression: a prospective study following mild traumatic brain injury, Medicine, Science \& the Law 41(2) (2001), 116-121.

[5] G.L. Iverson and L.M. Binder, Detecting exaggeration and malingering in neuropsychological assessment, Journal of Head Trauma Rehabilitation 15(2) (2000), 829-858. 
[6] P.S. Satz et al., Persistent Post-Concussive Syndrome: A proposed methodology and literature review to determine the effects, if any, of mild head and other bodily injury, Journal of Clinical \& Experimental Neuropsychology 21(5) (1999), 620-628.

[7] S.S. Dikmen and H.S. Levin, Methodological issues in the study of mild head injury, Journal of Head Trauma Rehabilitation 8 (1993), 30-37.

[8] R. Dacey et al., Relative effects of brain and non-brain injuries on neuropsychological and psychosocial outcome, Journal of Trauma 31(2) (1991), 217-222.

[9] S.S. Dikmen et al., Neuropsychological ourcome at 1-year post head injury, Neuropsychology 9 (1995), 80-90.

[10] S.S. Dikmen et al., One year psychosocial outcome in head injury, Journal of the International Neuropsychological Society 1 (1995), 67-77.

[11] D.B. Arciniegas and J.M. Silver, Regarding the search for a unified definition of mild traumatic brain injury, Brain Injury 15(7) (2001), 649-652.

[12] T.W. McAllister, Mild traumatic brain injury and the postconcussive syndrome, in: Neuropsychiatry of Traumatic Brain Injury, J. Silver, S. Yudofsky and R. Hales, eds, American Psychiatruc Press, Washington, DC, 1994, pp. 357-392.

[13] G. Teasdale and B. Jennett, Assessment of coma and impaired consciousness: a practical scale, Lancet 2 (1974), 81-84.

[14] T. Kay et al., Definition of Mild Traumatic Brain Injury, Jour nal of Head Trauma Rehabilitation 8(3) (1993), 86-87.

[15] N.S. Hinkeldey and J.D. Corrigan, The structure of head injured patients' neurobehavioral complaints: a preliminary study, Brain Inj 4 (1990), 115-133.

[16] W.W. McKinlay et al., The short term outcomes of severe blunt head injury as reported by relatives of the injured persons, $J$ Neurol Neurosurg Psychiatry 44 (1981), 527-533.

[17] A.H. Van Zomeron and W. Van Den Burg, Residual complaints of patients two years after severe head injury, J Neurol Neurosurg Psychiatry 48 (1985), 21-28.

[18] S. Deb, I. Lyons and C. Koutzoukis, Neuropsychiatric sequelae one year after a minor head injury, Journal of Neurology, Neurosurgery \& Psychiatry 65(6) (1998), 899-902.

[19] T.A. Gennarelli, Cerebral concussions and diffuse brain injuries, in: Head Injury, P.R. Cooper, ed., Williams and Wilkins, Baltimore, 1987, pp. 108-124.

[20] S.J. Peerless and N.W. Newcastle, Sheer injuries of the brain, Can Med Assoc J 96 (1967), 577-582.

[21] D.R. Oppenheimer, Microscopic lesions in the brain following head injury, Journal of Neurology, Neurosurgery \& Psychiatry 31(4) (1968), 299-306.

[22] P.C. Blumbergs et al., Staining of amyloid precursor protein to study axonal damage in mild head injury, Lancet 344(8929) (1994), 1055-1056.

[23] J.T. Povlishock and T.H. Coburn, Morhopathological change associated with mild head injury, in: Mild Head Injury, $\mathrm{H}$. Levin, H. Eisenberg and A. Benton, eds, Oxford University Press, New York, 1989, pp. 37-53.

[24] J.A. Jane, O. Steward and T. Gennarelli, Axonal degeneration induced by experimental noninvasive minor head injury, Journal of Neurosurgery 62(1) (1985), 96-100.

[25] J.T. Povlishock and C.W. Christman, The pathobiology of traumatically induced axonal injury in animals and humans: A review of current thoughts, Journal of Neurotrauma 12(4) (1995), 555-564.

[26] D.H. Williams, H.S. Levin and H.M. Eisenberg, Mild head injury classification, Neurosurgery 27(3) (1990), 422-428.
[27] P. Borczuk, Predictors of intracranial injury in patients with mild head trauma, Ann Emerg Med 25(6) (1995), 731-736.

28] E.C. Miller, J.F. Holmes and R.W. Derlet, Utilizing clinical factors to reduce head CT scan ordering for minor head trauma patients, J Emerg Med 15(4) (1997), 453-457.

[29] M.J. Haydel et al., Indications for computed tomography in patients with minor head injury [see comments], N Engl J Med 343(2) (2000), 100-105.

[30] S.R. Shackford et al., The clinical utility of computed tomographic scanning and neurologic examination in the management of patients with minor head injuries [see comments], $J$ Trauma 33(3) (1992), 385-394.

[31] S.C. Stein and S.E. Ross, Mild head injury: a plea for routine early CT scanning [see comments], J Trauma 33(1) (1992), $11-13$.

[32] W.K. Schynoll et al., A prospective study to identify highyield criteria associated with acute intracranial computed tomography findings in head-injured patients, American Journal of Emergency Medicine 11 (1993), 321-326.

[33] F.T. Harad and M.D. Kerstein, Inadequacy of bedside clinical indicators in identifying significant intracranial injury in trauma patients, J Trauma 32(3) (1992), 359-361; discussion 361-363.

[34] J. Van der Naalt et al., Computed tomography and magnetic resonance imaging in mild to moderate head injury: Early and late imaging related to outcome, Annals of Neurology 46 (1999), 70-78.

[35] S. McCullagh et al., Prediction of neuropsychiatric outcome following mild trauma brain injury: an examination of the Glasgow Coma Scale, Brain Injury 15(6) (2001), 489-497.

[36] T.W. McAllister et al., Neuroimaging findings in mild traumatic brain injury, Journal of Clinical \& Experimental Neuropsychology 23 (2001), 775-791.

[37] H.M. Abdel-Dayem et al., Changes in cerebral perfusion after acute head injury: comparison of CT with Tc-99m HM-PAO SPECT, Radiology 165 (1987), 221-226.

[38] R.H. Reid et al., Cerebral perfusion imaging with technetium99m HMPAO following cerebral trauma: Initial experience, Clinical Nuclear Medicine 15 (1990), 383-388.

[39] S.N. Roper et al., An analysis of cerebral blood flow in acute closed-head injury using Technetium-99m HMPAO SPECT and computed tomography, Journal of Nuclear Medicine 32 (1991), 1684-1687.

[40] M.R. Newton et al., A study comparing SPECT with CT and MRI after closed head injury, J Neurol Neurosurg Psychiatry 55(2) (1992), 92-94.

[41] S. Nagamachi et al., A comparative study of 123I-IMP SPET and CT in the investigation of chronic-stage head trauma patients, Nuclear Medicine Communications 16(1) (1995), 1725.

[42] A. Jacobs et al., Prospective evaluation of technetium-99mHMPAO SPECT in mild and moderate traumatic brain injury, Journal of Nuclear Medicine 35 (1994), 942-947.

[43] M.S. Choksey et al., 99TCm-HMPAO SPECT studies in traumatic intracerebral haematoma, J Neurol Neurosurg Psychiatry 54(1) (1991), 6-11.

[44] I. Silverman et al., SPECT in patients with cortical visual loss, Journal of Nuclear Medicine 34 (1993), 1447-1451.

[45] T.W. Langfitt et al., Computerized tomography, magnetic resonance imaging, and positron emission tomography in the study of brain trauma. Preliminary observations, J Neurosurg 64(5) (1986), 760-767.

[46] A. Alavi, Functional and anatomical studies of head injury, Journal of Neuropsychiatry 1 (1989), S45-S50. 
[47] J.K. George et al., Metabolic (PET) correlates of anatomic lesions (CT/MRI) produced by head trauma, Journal of Nuclear Medicine 30 (1989), 802.

[48] R.M. Ruff et al., Computerized tomography, neuropsychology, and positron emission tomography in the evaluation of head injury, Neuropsychiatry, Neuropsychology and Behavioral Neurology 2 (1989), 103-123.

[49] A. Alavi and A.B. Newberg, Metabolic consequences of acute brain trauma: Is there a role for PET? Journal of Nuclear Medicine 37 (1996), 1170-1172.

[50] M.S. Humayun et al., Local cerebral glucose abnormalities in mild closed head injured patients with cognitive impairments, Nucl Med Commun 10(5) (1989), 335-344.

[51] R.M. Ruff et al., Recovery of memory after mild head injury: a three center study, in: Mild Head injury, H.S. Levin, H.M. Eisenberg and A.L. Benton, eds, Oxford University Press, New York, 1989, pp. 176-188.

[52] R.M. Ruff et al., Selected cases of poor outcome following a minor brain trauma: comparing neuropsychological and positron emission tomography assessment, Brain Inj 8(4) (1994), 297-308.

[53] H. Gross et al., Local cerebral glucose metabolism in patients with long-term behavioral and cognitive deficits following mild traumatic brain injury, Journal of Neuropsychiatry and Clinical Neurosciences 8 (1996), 324-334.

[54] T.W. McAllister et al., Differences in working memoryassociated brain activation one month after mild traumatic brain injury: an fMRI study, Neurology 53 (1999), 1300-1309.

[55] T.W. McAllister et al., Differential working memory load effects after mild traumatic brain injury, Neuroimage 14 (2001), 1004-1012.

[56] K.M. Cecil et al., Proton magnetic resonance spectroscopy for detection of axonal injury in the splenium of the corpus callosum of brain-injured patients, Journal of Neurosurgery 88 (1998), 795-801.

[57] M.R. Garnett et al., Evidence for cellular damage in normalappearing white matter correlates with injury severity in patients following traumatic brain injury: A magnetic resonance spectroscopy study, Brain 123 (2000), 1403-1409.

[58] W.M. Brooks et al., Metabolic and cognitive response to human traumatic brain injury: a quantitative proton magnetic resonance study, Journal of Neurotrauma 17(8) (2000), 629640.

[59] M.R. Garnett et al., Altered cellular metabolism following traumatic brain injury: a magnetic resonance spectroscopy study, Journal of Neurotrauma 18(3) (2001), 231-240.

[60] M. Gaetz and D.M. Bernstein, The current status of electrophysiologic procedures for the assessment of mild traumatic brain injury, Journal of Head Trauma Rehabilitation 16(4) (2001), 386-405.

[61] R. Schoenhuber and M. Gentilini, Neurophysiological assessment of mild head injury, in: Mild Head Injury, H.S. Levin, H.M. Eisenberg and A.L. Benton, eds, Oxford University Press, New York, 1989, pp. 142-150.

[62] B. Voller et al., Neuropsychological, MRI and EEG findings after very mild traumatic brain injury, Brain Injury 13(10) (1999), 821-827.

[63] H.J. Garber et al., Clinical use of topographic brain electrical activity mapping in psychiatry, J Clin Psychiatry 50 (1989), 205-211.

[64] R.W. Thatcher et al., EEG discriminant analyses of mild head trauma, Electroencephanography and Clinical Neurophysiology 73 (1989), 93-106.
[65] R.W. Thatcher et al., Biophysical linkage between MRI and EEG amplitude in closed head injury, Neuroimage 7 (1998), 352-367.

[66] R.W. Thatcher et al., Biophysical linkage between MRI and EEG coherence in closed head injury, Neuroimage 8 (1998), 307-326.

[67] R.W. Thatcher et al., An EEG severity index of traumatic brain injury, Journal of Neuropsychiatry \& Clinical Neurosciences 13 (2001), 77-87.

[68] R. Schoenhuber and M. Gentilini, Auditory brain stem responses in the prognosis of late postconcussional symptoms and neuropsychological dysfunction after minor head injury, Neurosurgery 19 (1986), 532-534.

[69] R. Pratrap-Chand, M. Sinniah and F.A. Salem, Cognitive evoked potential (P300): a metric for cerebral concussion, Acta Neurol Scand 78 (1988), 185-189.

[70] M.R. Abd Al-Hady et al., Audiological findings following head trauma, J Laryngol Otol 104 (1990), 927-936.

[71] M.E. Drake, Jr., S.J. Weate and S.A. Newell, Auditory evoked potentials in postconcussive syndrome, Electromyography \& Clinical Neurophysiology 36(8) (1996), 457-462.

[72] J.F. Soustiel et al., Trigeminal and auditory evoked responses in minor head injuries and post-concussion syndrome, Brain Injury 9(8) (1995), 805-813.

[73] S. Freed and L.F. Hellerstein, Visual electrodiagnostic findings in mild traumatic brain injury, Brain Injury 11(1) (1997), 2536.

[74] M. Gaetz, D. Goodman and H. Weinberg, Electrophysiological evidence for the cumulative effects of concussion, Brain Injury 14(12) (2000), 1077-1088.

[75] M. Gaetz and H. Weinberg, Electrophysiological indices of persistent post-concussion symptoms, Brain Injury 14(9) (2000), 815-832.

[76] P. Papathanasopoulos et al., Pattern reversal visual evoked potentials in minor head injury, European Neurology 34 (1994), 268-271.

[77] D.B. Arciniegas et al., Attention and memory dysfunction after traumatic brain injury: cholinergic mechanisms, sensory gating, and a hypothesis for further investigation, Brain Injury 12 (1999), 1-13.

[78] D.B. Arciniegas et al., Impaired auditory gating and P50 nonsuppression following traumatic brain injury, Journal of Neuropsychiatry \& Clinical Neurosciences 12(1) (2000), 77-85.

[79] D.B. Arciniegas et al., Reduced hippocampal volume in association with P50 nonsuppression following traumatic brain injury, Journal of Neuropsychiatry \& Clinical Neurosciences 13(2) (2001), 213-221.

[80] D.B. Arciniegas et al., Normalization of P50 physiology by donepezil hydrochloride in traumatic brain injury patients, Journal of Neuropsychiatry and Clinical Neuroscience 13(1) (2001), 140.

[81] H.S. Levin et al., Neurobehavioral outcome following minor head injury: a three-center study, Journal of Neurosurgery 66(2) (1987), 234-243.

[82] D.T. Stuss et al., Reaction time after head injury: fatigue, divided and focused attention, and consistency of performance, Journal of Neurology, Neurosurgery \& Psychiatry 52(6) (1989), 742-748.

[83] R.M. Ruff et al., Recovery of memory after mild head injury: a three center study, in: Mild Head Injury, H. Levin, H. Eisenberg and A. Benton, eds, Oxford University Press, New York, 1989, pp. 176-188. 
[84] T.M. McMillan and E.E. Glucksman, The neuropsychology of moderate head injury, Journal of Neurology, Neurosurgery \& Psychiatry 50(4) (1987), 393-397.

[85] D. Gronwall, Cumulative and persisting effects of concussion on attention and cognition, in: Mild Head Injury, H. Levin, $\mathrm{H}$. Eisenberg and A. Benton, eds, Oxford University Press, New York, 1989, pp. 153-162.

[86] M. Gentilini, P. Nichelli and R. Schoenhuber, Assessment of attention in mild head injury, in: Mild Head Injury, H. Levin, H. Eisenberg and A. Benton, eds, Oxford University Press, New York, 1989, pp. 163-175.

[87] S. Dikmen et al., Neuropsychologic outcome at one-month postinjury, Archives of Physical Medicine \& Rehabilitation 67(8) (1986), 507-513.

[88] N. Bohnen, A. Twijnstra and J. Jolles, Persistence of postconcussional symptoms in uncomplicated, mildly head-injured patients: a prospective cohort study, Neuropsychiatry, Neuropsychology, \& Behavioral Neurology 6 (1993), 193-200.

[89] R.W. Rimel et al., Disability caused by minor head injury, Neurosurgery 9(3) (1981), 221-228.

[90] T. Ingebrigtsen et al., Quantification of post-concussion symptoms 3 months after minor head injury in 100 consecutive patients, Journal of Neurology 245(9) (1998), 609-612.

[91] D.P. Goldberg and V.F. Hebber, A scaled version of the general health questionnaire, Psychological Medicine 9 (1979), 139145.

[92] W. Alves, S.N. Macciocchi and J.T. Barth, Postconcussive symptoms after uncomplicated mild head injury, Journal of Head Trauma Rehabilitation 8(3) (Sep 1993), 48-59.

[93] S. Dikmen, A. McLean and N. Temkin, Neuropsychological and psychosocial consequences of minor head injury, Journal of Neurology, Neurosurgery \& Psychiatry 49(11) (1986), 1227-1232.

[94] L.M. Binder, M.L. Rohling and J. Larrabee, A review of mild head trauma. Part I: Meta-analytic review of neuropsychological studies, Journal of Clinical \& Experimental Neuropsychology 19(3) (1997), 421-431.

[95] L.M. Binder, A Review of Mild Head Trauma 2: Clinical Implications [Review], Journal of Clinical \& Experimental NeuropsychologyInternational Journal of Radiation Applications \& Instrumentation - Part B, Nuclear Medicine \& Biology 19(3) (1997), 432-457.

[96] B.E. Leininger et al., Neuropsychological deficits in symptomatic minor head injury patients after concussion and mild concussion [see comments], Journal of Neurology, Neurosurgery \& Psychiatry 53(4) (1990), 293-296.

[97] T.J. Guilmette and D. Rasile, Sensitivity, specificity and diagnostic accuracy of three verbal memory measures in the assessment of mild brain injury, Neuropsychology 9 (1995), 338-344.

[98] B. Jennett, Assessment of the severity of head injury, Journal of Neurology, Neurosurgery \& Psychiatry 39 (1976), 647-655.

[99] S. Crawford, F.J. Wenden and D.T. Wade, The Rivermead Head Injury Follow Up Questionnaire: A study of a new rating scale and other measures to evaluate outcome after head injury, Journal of Neurology, Neurosurgery \& Psychiatry 60 (1996), 510-514.

[100] M.S. Keshavan, S.M. Channabasavanna and G.N.N. Reddy, Post-traumatic psychiatric disturbances: patterns and predictors of outcome, Br J Psychiatry 138 (1981), 157-160.

[101] H. Merskey and J.M. Woodforde, Psychiatric sequelae of minor head injury, Brain 95 (1972), 521-528.

[102] W.H. Rutherford, Postconcussive symptoms: relationship to acute neurological indices, individual differences, and circum- stances of injury, in: Mild Head injury, H.S. Levin, H.M. Eisenberg and A.L. Benton, eds, Oxford University Press, New York, 1989, pp. 217-228.

[103] J.E. Meyers and M. Volbrecht, Detection of malingerers using the Rey Complex Figure and Recognition Trial, Applied Neuropsychology 6(4) (1999), 201-207.

[104] M.P. Alexander, Mild traumatic brain injury: pathophysiology, natural history, and clinical management [see comments], Neurology 45(7) (1995), 1253-1260.

[105] K.E. Goethe and H.S. Levin, Behavioral manifestation during the early and long-term stages of recovery after closed head injury, Psychiatric Annals 14 (1984), 540-546.

[106] D. Leigh, Psychiatric aspects of head injury, Jounal of Clinical and Experimental Psychiatry 40 (1979), 21-33.

[107] W.A. Lishman, The psychiatric sequelae of head injury: a review, Psychol Med 3 (1973), 304-318.

[108] W.A. Lishman, Physiogenesis and psychogenesis in the "post-concussional syndrome", Brithish Journal of Psychiatry 153 (1988), 460-469.

[109] N.S. King, Emotional, neuropsychological, and organic factors: their use in the prediction of persisting postconcussion symptoms after moderate and mild head injuries, Journal of Neurology, Neurosurgery \& Psychiatry 61(1) (1996), 75-81.

[110] N.S. King et al., Early prediction of persisting postconcussion symptoms following mild and moderate head injuries, British Journal of Clinical Psychology 38(1) (1999), $15-25$.

[111] T. Kay, Neuropsychological Diagnosis: Disentangling the multiple determinants of functional disability after mild traumatic brain injury, in: Physical Medicine and Rehabilitation: State of the Art Reviews, Hanley \& Belfus, Inc., Philadelphia, 1992, pp. 109-127.

[112] D.B. Arciniegas, J. Topkoff and J.M. Silver, Neuropsychiatric aspects of traumatic brain injury, Current Treatment Options in Neurology 2(2) (2000), 169-186.

[113] R. van Reekum, T. Cohen and J. Wong, Can traumatic brain injury cause psychiatric disorders? Journal of Neuropsychiatry and Clinical Neuroscience 12 (2000), 316-327.

[114] C.R. Busch and H.P. Alpern, Depression after mild traumatic brain injury: a review of current research, Neuropsychology Review 8(2) (1998), 95-108.

[115] J.D. Gfeller, J.T. Chibnall and P.N. Duckro, Postconcussion symptoms and cognitive functioning in postraumatic headache patients, Headache 34 (1994), 503-507.

[116] T.W. McAllister and L.A. Flashman, Mild brain injury and mood disorders: causal connection, assessment, and treatment, in: The Evaluation and Treatment of Mild Traumatic Brain Injury, N.R. Varney and R.J. Roberts, eds, Lawrence Erlbaum Associates, Mahwah, NJ, 1999, pp. 347-373.

[117] J.M. Burke, C.L. Imhoff and J.M. Kerrigan, MMPI correlates among post-acute TBI patients, Brain Inj 4 (1990), 223-231.

[118] D.J. Fordyce, J.R. Roueche and G.P. Prigatano, Enhanced emotional reactions in chronic head trauma patients, J Neurol Neurosurg Psychiatry 46 (1983), 620-624.

[119] J.R. Fann et al., Psychiatric disorders and functional disability in outpatients with traumatic brain injuries, American Journal of Psychiatry 152(10) (1995), 1493-1499.

[120] J.P. Federoff et al., Depression in patients with acute traumatic brain injury, American Journal of Psychiatry 149 (1992), 918-923.

[121] R.E. Jorge et al., Comparison between acute- and delayedonset depression follwoing traumatic brain injury, Journal of Neuropsychiatry and Clinical Neuroscience 5 (1993), 43-49. 
[122] R.E. Jorge et al., Depression following traumatic brain injury: A 1 year longitudinal study, Journal of Affective Disorders 27 (1993), 233-243

[123] R. Schoenhuber and M. Gentilini, Anxiety and depression after mild head injury: a case control study, J Neurol Neurosurg Psychiatry 51 (1988), 722-724.

[124] M. Mobayed and T.G. Dinan, Buspirone/prolactin response in post head injury depression, J Affect Disord 19 (1990), 237-241.

[125] M.A. Hamilton, R.P. Smith and G.W. Bridge, The Leeds scale for the self-assessment of anxiety and depression, $\mathrm{Br} J$ Psychiatry 128 (1976), 156-165.

[126] A.S. Saran, Depression after mild closed head injury: role of dexametharone suppression test and antidepressants, J Clin Psychiatry 1985 (1985), 335-338.

[127] R.E. Jorge et al., Influence of major depression on 1-year outcome in patients with traumatic brain injury, Journal of Neurosurgery 81 (1994), 726-733.

[128] L.M. Binder, Persisting symptoms after mild head injury: a review of the postconcussive syndrome, Journal of Clinical \& Experimental Neuropsychology 8(4) (1986), 323-346.

[129] R.A. Mayou, J. Black and B. Bryant, Unconsciousness, amnesia and psychiatric symptoms following road traffic accident injury, British Journal of Psychiatry 177 (2000), 540-545.

[130] R.A. Bryant and A.G. Harvey, Relationship between acute stress disorder and posttraumatic stress disorder following mild traumatic brain injury, American Journal of Psychiatry 155(5) (1998), 625-629.

[131] R.A. Bryant and A.G. Harvey, Postconcussive symptoms and posttraumatic stress disorder after mild traumatic brain injury, Journal of Nervous \& Mental Disease 187(5) (1999), 302305.

[132] R.A. Bryant and A.G. Harvey, The influence of traumatic brain injury on acute stress disorder and post-traumatic stress disorder following motor vehicle accidents, Brain Injury 13(1) (1999), 15-22.

[133] A.G. Harvey and R.A. Bryant, Acute stress disorder after mild traumatic brain injury, Journal of Nervous \& Mental Disease 186(6) (1998), 333-337.

[134] A.G. Harvey and R.A. Bryant, Predictors of acute stress following mild traumatic brain injury, Brain Injury 12(2) (1998), $147-154$.

[135] A.G. Harvey and R.A. Bryant, Two-year prospective evaluation of the relationship between acute stress disorder and posttraumatic stress disorder following mild traumatic brain injury, American Journal of Psychiatry 157(4) (2000), 626-628.

[136] V. Rao and P. Rollings, Sleep disturbances following traumatic brain injury, Current Treatment Options in Neurology (in press).

[137] A. McLean et al., Psychosocial functioning at one month after head injury, Neurosurgery 14 (1984), 393-399.

[138] F.R. Sparadeo, D. Strauss and J.T. Bartels, The incidence, impact, and treatment of substance abuse in head trauma rehabilitation, J Head Traum Rehabil 5 (1990), 108.

[139] G. Davidoff et al., Closed head injury in spinal cord injured patients: retrospective study of loss of consciousness and posttraumatic amnesia, Arch Phys Med Rehabil 66 (1985), 41-43.

[140] L.J. Miller and W. Mittenberg, Brief cognitive behavioral interventions in mild traumatic brain injury, Applied Neuropsychology 5 (1998), 172-183.

[141] R. Kelly, The post-traumatic syndrome: an iatrogenic disease, Forensic Science 6 (1975), 17-24.

[142] W. Minderhoud et al., Treatment of minor head injuries, Clinical Neurology and Neurosurgery 82 (1980), 127-140.
[143] J.M. Minderhoud et al., Treatmetn of minor head injuries, Clinical Neurology and Neurosurgery 82 (1997), 127-140.

[144] W. Mittenberg et al., Cognitive-behavioral prevention of postconcussion syndrome, Archives of Clinical Neuropsychology 11 (1996), 139-145.

[145] C. Paniak et al., A randomized trial of two treatments for mild traumatic brain injury, Brain Injury 12(12) (1998), 10111023.

[146] D.T. Wade et al., Does routine follow up after head injury help? A randomised controlled trial, Journal of Neurology, Neurosurgery \& Psychiatry 62(5) (1997), 478-484.

[147] D.T. Wade et al., Routine follow up after head injury: a second randomised controlled trial, Journal of Neurology, Neurosurgery \& Psychiatry 65(2) (1998), 177-183.

[148] P. Wrightson, Management of disability and rehabilitation services after mild head injury, in: Mild Head Injury, H.S. Levin, H.M. Eisenberg and A.L. Benton, eds, Oxford University Press, New York, 1989, pp. 245-256.

[149] D.N. Cope, Psychopharmacologic consideration in the treatment of traumatic brain injury, Journal of Head Trauma Rehabilitation 2(4) (1987), 5.

[150] C.T. Gualtieri, Pharmacotherapy and the neurobehavioral sequelae of traumatic brain injury, Brain Inj 2 (1988), 101-129.

[151] T.W. McAllister, Neuropsychiatric sequelae of head injuries, Psychiatric Clinics of North America 15(2) (1992), 395-413.

[152] J.M. Silver, R.E. Hales and S.C. Yudofsky, Neuropsychiatric aspects of traumatic brain injury, in: The American Psychiatric Press Textbook of Neuropsychiatry, S.C. Yudofsky and R.E. Hales, eds, American Psychiatric Press, Inc., Washington, DC, 1992, pp. 179-190.

[153] T.W. McAllister, Mixed neurologic and psychiatric disorders: Pharmacological issues, Compr Psychiatry 33(5) (1992), 296304.

[154] J.R. Fann, J.M. Uomoto and W.J. Katon, Sertraline in the treatment of major depression following mild traumatic brain injury, Journal of Neuropsychiatry \& Clinical Neurosciences 12(2) (2000), 226-232.

[155] T.W. McAllister and T.R.P. Price, Depression in the brain injured:phenomenology and treatment, in: Depression: New Directions in Theory, Research, and Practice, N.S. Endler and C.D. McCann, eds, Wall and Emerson, Toronto, Canada, 1990, pp. 361-387.

[156] S. Shukla et al., Mania following head trauma, Am J Psychiatry 144 (1987), 93-96.

[157] J.M. Silver, S.C. Yudofsky and R.E. Hales, Depression in traumatic brain injury, Neuropsychiatry, Neuropsychology, and Behavioral Neurology 4 (1991), 12-23.

[158] K. Cicerone et al., Evidence-based cognitive rehabilitation recommendations for clinical practice, Archives of Physical Medicine \& Rehabilitation 8 (2000), 1596-1615.

[159] A.F.T. Arnsten, Catecholamine modulation of prefrontal cortical cognitive function, Trends in Cognitive Sciences 2(11) (1998), 436-447.

[160] M. Luciana et al., Facilitation of working memory in humans by a D2 dopamine receptor agonist, Journal of Cognitive Neuroscience 4(1) (1992), 58-68.

[161] M. Luciana and P.F. Collins, Dopaminergic modulation of working memory for spatial but not object cues in normal humans, Journal of Cognitive Neuroscience 9 (1997), 330347.

[162] R. Elliot et al., Effects of Methylphenidate on spatial working memory and planning in healthy young adults, Psychopharmacology 131 (1997), 196-206. 
[163] S. McDowell, J. Whyte and M. D'Esposito, Differential effect of a dopaminergic agonist on prefrontal function in traumatic brain injury patients, Brain 121 (1998), 115-1164.

[164] M.B. Glenn, Methylphenidate for cognitive and behavioral dysfunction after traumatic brain injury, J Head Trauma Rehabilitation 13(5) (1998), 87-90.

[165] C.T. Gualtieri and R.W. Evans, Stimulant treatment for the neurobehavioral sequelae of traumatic brain injury, Brain Injury 2 (1988), 273-290.

[166] C.T. Gualtieri and R.W. Evans, Stimulant treatment for the neurobehavioural sequelae of traumatic brain injury, Brain Injury 2(4) (1988), 273-290.

[167] C.T. Gualtieri et al., Amantadine: a new clinical profile for traumatic brain injury, Clin Neuropharmacol 12 (1989), 258270.

[168] J. Whyte et al., Effects of methylphenidate on attentional function after traumatic brain injury: A randomized, placebocontrolled trial, American Journal of Physical Rehabilitation 76(6) (1997), 440-450.

[169] M.L. Crismon et al., The effect of bromocriptine on speech dysfunction in patients with diffuse brain injury (akinetic mutism), Clinical Neuropharmacology 11(5) (1988), 462466.

[170] B.H. Dobkin and R. Hanlon, Dopamine agonist treatment of anterograde amnesia from a mediobasal forebrain injury, Annals of Neurology 33 (1993), 313-316.

[171] D.L. Kaelin, D.X. Cifu and B. Matthies, Methylphenidate effect on attention deficit in the acutely brain-injured adult, Arch Phys Med Rehabil 77 (1996), 6-9.

[172] S. Lal, C.P. Merbtiz and J.C. Grip, Modification of function in head-injured patients with Sinemet, Brain Injury 2(3) (1988), 225-233.

[173] G.F. Mooney and I.J. Haas, Effect of methylphenidate on brain injury-related anger, Arch Phys Med Rehabil 74 (1993), 153-160.

[174] P.M. Plenger et al., Subacute methylphenidate treatment for moderate to moderately severe traumatic brain injury: A preliminary double-blind placebo-controlled study, Arch. Phys. Med. Rehabil. 77 (1996), 536-540.

[175] J.H. Powell et al., Motivational deficits after brain injury: effects of bromocriptine in 11 patients, Journal of Neurology, Neurosurgery, Psychiatry 60 (1996), 416-421.

[176] A.F. Arnsten et al., Noradrenergic influences on prefrontal cortical cognitive function: opposing actions at postjunctional alpha 1 versus alpha 2-adrenergic receptors, Advances in Pharmacology (New York) 42 (1998), 764-767.

[177] R.T. Bartus, D. Fleming and H.R. Johnson, Aging in the rhesus monkey: debilitation effects on short-term memory, Journal of Gerontology 33 (1978), 858-871.

[178] T. Brozoski et al., Cognitive deficit caused by regional depletion of dopamine in prefrontal cortex of rhesus monkey, Science 205 (1979), 929-931.

[179] J.X. Cai, L. Xu and X. Hu, Reserpine impairs spatial workingmemory performance in monkeys: reversal by the alpha-2 adrenergic agonist clonidine, Brain Research 614 (1993), 191196.

[180] V. Luine, D. Bowling and M. Hearns, Spatial memory deficits in aged rats: contributions of monoaminergic systems, Brain Research 537 (1990), 271-278.

[181] J.C. Steere and A.F. Arnsten, The alpha-2A noradrenergic receptor agonist guanfacine improves visual object discrimina- tion reversal performance in aged rhesus monkeys, Behavioral Neuroscience 111(5) (1997), 883-891.

[182] H. Tanila, P. Rama and S. Carlson, The effects of prefrontal intracortical microinjections of an alpha-2 agonist, alpha- 2 antagonist and lidocaine on the delayed alternation performance of aged rats, Brain Research Bulletin 40(2) (1996), 117-119.

[183] A.F. Arnsten et al., Alpha-1 noradrenergic receptor stimulation impairs prefrontal cortical cognitive function, Biological Psychiatry 45(1) (1999), 26-31.

[184] P. Jakala et al., Guanfacine, but not clonidine, improves planning and working memory performance in humans, Neuropsychopharmacology 20(5) (1999), 460-470.

[185] P. Jakala et al., Guanfacine and clonidine, alpha 2-agonists, improve paired associates learning, but not delayed matching to sample, in humans, Neuropsychopharmacology 20(2) (1999), 119-130.

[186] C.E. Dixon et al., Traumatic brain injury reduces hippocampal high affinity $[3 \mathrm{H}]$ choline uptake but not extracellular choline levels in rats, Neuroscience Letters 180 (1994), 127130.

187] M.M. DeAngelis, R.L. Hayes and B.G. Lyeth, Traumatic brain injury causes a decrease in M2 muscarinic cholinergic receptor binding in the rat brain, Brain Research 653 (1994), 39-44.

[188] A. Saija et al., The effects of scopolamine and traumatic brain injury on central cholinergic neurons, Journal of Neurotrauma 5 (1988), 161-170.

[189] I. Murdoch et al., Cortical cholinergic dysfunction after human head injury, Journal of Neurotrauma 15 (1998), 295-305.

[190] D. Dewar and D.I. Graham, Depletion of choline acetyltransferase but preservation of M1 and M2 muscarinic receptor binding sites temporal cortex following head injury: A preliminary human postmorten study, Journal of Neurotrauma 13 (1996), 181-187.

[191] T.G. Aigner, Pharmacology of memory: cholinergicglutamatergic interactions, Current Opinion in Neurobiology 5 (1995), 155-160.

[192] U.J. Bogdanovitch, G.J. Bazarevitch and A.L. Kirillov, The use of cholinesterase in severe head injury, Resuscitation 4 (1975), 139-141.

[193] D.D. Cardenas et al., Oral physostigmine and impaired memory in adults with brain injury, Brain Injury 12 (1994), 77-80.

[194] E. Goldberg et al., Selective effects of cholinergic treatment of verbal memory in posttraumatic amnesia, Journal of Clinical Neuropsychology 4 (1982), 219-234.

[195] H.S. Levin et al., Effects of oral physostigmine and lecithin on memory and attention in closed head-injured patients, Centeral Nervous System Trauma 3 (1986), 333-342.

[196] P. Eames and A. Sutton, Protracted post-traumatic confusional state treated with physostigmine, Brain Injury 9 (1995), 729-734.

[197] J.P. Tayerni, G. Seliger and S.W. Lichtman, Donepezil mediated memory improvement in traumatic brain injury dufing post acute rehabilitation, Brain Injury 12 (1998), 77-80.

[198] F.J. Whelan, M.S. Walker and S.K. Schultz, Donepezil in the treatment of cognitive dysfunction associated with traumatic brain injury, Annals of Clinical Psychiatry 12 (2000), 131135.

[199] T.J. Speech et al., A double-blind controlled study of methylphenidate treatment in closed head injury, Brain Injury 7 (1993), 333-338. 